\title{
A UNIQUENESS THEOREM FOR THE LEGENDRE AND HERMITE POLYNOMIALS*
}

BY

\section{K. P. WILLIAMS}

1. If we replace $y$ in the expansion of $(1+y)^{-\nu}$ by $2 x z+z^{2}$, the coefficient of $z^{n}$ will, when $x$ is replaced by $-x$, be the generalized polynomial $L_{n}^{\prime}(x)$ of Legendre. It is also easy to show that the Hermitian polynomial $H_{n}(x)$, usually defined by

$$
e^{x^{2}} \frac{d^{n}}{d x^{n}} e^{-x^{2}}=H_{n}(x),
$$

is the coefficient of $z^{n} / n$ ! in the series obtained on replacing $y$ in the expansion of $e^{-y}$ by the same expression $2 x z+z^{2}$. Furthermore, there is a simple recursion formula between three successive Legrendre polynomials and between three successive Hermitian polynomials. These facts suggest the following problem.

Let

and put

$$
\varphi(y)=a_{0}+a_{1} y+\frac{a_{2}}{2 !} y^{2}+\frac{a_{3}}{3 !} y^{3}+\cdots
$$

$$
\varphi\left(2 x z+z^{2}\right)=P_{0}+P_{1}(x) z+P_{z}(x) z^{2}+\cdots .
$$

To what extent is the generating function $\varphi(y)$ determined if it is known that a simple recursion relation exists between three of the successive polynomials $P_{0}, P_{1}(x), P_{2}(x), \ldots$ ? We shall find that the generalized Legendre polynomials and those of Hermite possess a certain uniqueness in this regard.

2. We have

$$
P_{n}(x)=\left.\frac{1}{n !} \quad \frac{d^{n}}{d z^{n}} \varphi\left(2 x z+z^{2}\right)\right|_{z=0} .
$$

When we make use of the formula for the $u$ th derivative of a function of a function given by Faà de Bruno, $t$ we find without difficulty

$$
P_{n}(x)=\sum \frac{a_{n-j}}{i ! j !}(2 x)^{i},
$$

Presented to the Suciety, October 25, 1924.

$\uparrow$ Quarterly Journal of Mathematics, vol. 1, p. 359. 
where the summation extends to all values of $i$ and $j$ subject to the relation

$$
i+2, j=n \text {. }
$$

When developed, the expression is

$$
P_{n}(x)=\frac{a_{n}}{n !}(2 x)^{n}+\frac{a_{n-1}}{(n-2) !}(2 x)^{n-2}+\frac{a_{n-2}}{(n-4) ! 2 !}(2 x)^{n-4}+\cdots
$$

It is seen that while $P_{n}$ is an even or an odd function, the coefficients of the generating function that enter into it form a certain consecutive group, a fact which has important consequences.

3. Let us denote by $A_{n}^{m}$ the term in $P_{n}(x)$ that is of degree $m$ in $x$. We see that

$$
\begin{aligned}
A_{n}^{n-2 j} & =\frac{a_{n-j}}{(n-2 j) ! j !}(2 x)^{n-2 j}, \\
A_{n+1}^{n-2 j-1} & =\frac{a_{n-j}}{(n-2 j-1) !(j+1) !}(2 x)^{n-2 j-1}, \\
A_{n+2}^{n-2 j} & =\frac{a_{n-j+1}}{(n-2 j) !(j+1) !}(2 x)^{n-2 j,}
\end{aligned}
$$

the expressions being valid for $j=-1,0,1,2, \ldots$ if we agree that $A_{n}^{m}=0$, when $m>n$. The notable fact is that $A_{n}^{n-2 j}, A_{n+1}^{n-2 j-1}$ both contain $a_{n-j}$, but $A_{n+2}^{n-2 j}$ contains $a_{n-j+1}$.

Let $k$ and $l$ be multipliers, which we shall assume to be polynomials in $n$ to be determined; then

$$
2 x l A_{n+1}^{n-2 j-1}+k A_{n}^{n-2 j}=[l n+(k-2 l) j+k] \frac{a_{n-j}}{a_{n-j+1}} A_{n+2}^{n-2 j},
$$

a formula valid for $j=0,1,2, \ldots$. Let

$$
\psi(j)=l n+(k-2 l) j+k .
$$

We see that

$$
\psi(--1)=(n+2) l,
$$

and when $n$ is even, that

$$
\psi\left(\frac{n}{2}\right)==\left(\frac{n}{2}+1\right) k .
$$


This shows that, $h$ being another polynomial in $n$ to be determined,

$$
h P_{n+2}-2 x l P_{n+1}-k P_{n}=\sum_{j=-1}^{n^{\prime}}\left\{h-\psi(j) \frac{a_{n-j}}{a_{n-j+1}}\right\} A_{n+2}^{n-2 j},
$$

where $n^{\prime}=n / 2$, if $n$ is even, and $n^{\prime}=(n-1) / 2$ if $n$ is odd.

4. We see from the last expression what must be the character of the recursion relation,* and that for it to exist we must have

$$
a_{n+1}=\varphi(n) a_{n}
$$

where $\varphi(n)$ is a polynomial in $n$. In order that the summation on the right vanish, it is necessary that

$$
\psi(j)=\varphi(n-j) \theta(n),
$$

$\theta(n)$ being a polynomial in $n$. The polynomial $h(n)$ is then given at once by

$$
h(n)=\theta(n)
$$

It is easy to determine $l$ and $k$, so that $\psi(j)$ will have the desired form. Since $\varphi(n-j)$ is of the same degree in $j$ that $\varphi(n)$ is in $n$, and since $\psi(j)$ is linear in $j$, we see that $\varphi(n)$ must be linear in $n$.

Put

$$
\varphi(n)=\alpha n+\beta \text {. }
$$

Then

$$
l n+(k-2 l) j+k=(\alpha n-\alpha j+\beta) \theta(n) .
$$

This is to be an identity in both $n$ and $j$. Put $j=-1$, and we find

$$
(n+2) l=(\alpha n+\alpha+\beta) \theta(n) .
$$

Since $\alpha$ and $\beta$ are arbitrary it follows that $\theta(n)$ must contain $n+2$ as a factor, and

$$
l=(\alpha n+\alpha+\beta) \frac{\theta(n)}{n+2}
$$

* It is evident that a linear recursion relation will not exist unless the factor $2 x$ is introduced as in the middle term above. 
It follows then at once that

$$
k=(\alpha n+2 \beta) \frac{\theta(n)}{n+2} .
$$

No loss of generality results from putting

$$
h=\theta(n)=(n+2), \quad l=(\kappa n+\kappa+\beta), \quad l i=(\alpha n+2 \beta) .
$$

The polynomials will therefore have the recursion relation

$$
(n+2) P_{n \dashv 2}(x)-2 x(\alpha n+\alpha+\beta) P_{n+1}(x)-(u n+2 \beta) P_{n}(x)=0,
$$

if

$$
a_{n+1}=(\alpha n+\beta) a_{n}
$$

Taking $a_{0}=1$, we have for generating function

$$
\varphi(y)=F\left(a, \frac{\beta}{\alpha}, a, \alpha y\right)=(1-\alpha y)^{-\beta / \alpha}, \text { if } a \neq 0
$$

where $F$ represents the hypergeometric function, and

$$
\varphi(y)=e^{\beta y}, \text { if } a=0 \text {. }
$$

These then are the only types of generating function that will give a recursion relation, with the conditions that $h, l$, and $k$ are polynomials in $n .^{*}$

๖. A further remark might be made about the case $\alpha \neq 0$.

We have

$$
2 \varphi^{\prime}\left(2 x z+z^{2}\right)=P_{1}^{\prime}(x)+P_{2}^{\prime}(x) z+P_{3}^{\prime}(x) z^{2}+\cdots .
$$

Also we find

$$
\varphi^{\prime}(y)(1-\varkappa y)=\beta \cdot \varphi(y),
$$

and can then deduce

$$
P_{n+2}^{\prime}(x)-2 \alpha x P_{n+1}^{\prime}(x)-\varkappa P_{n}^{\prime}(x)=2 \beta P_{n+1}(x) \text {. }
$$

When this is combined with the recursion formula we have

$$
x P_{n+1}^{\prime}(x)+P_{n}^{\prime}(x)=(n+1) P_{n+1}(x),
$$

\footnotetext{
* It would evidently be no more general to take $h, l, k$ rational in $n$.
} 
a relation independent of $\alpha$ and $\beta$. From this and the recursion relation we can obtain

$$
\left(1+\alpha x^{2}\right) P_{n}^{\prime \prime}+(\alpha+2 \beta) x P_{n}^{\prime}-n(\alpha n+2 \beta) P_{n}=0 .
$$

Now the differential equation

$$
\left(1+\alpha x^{2}\right) \frac{d^{2} y}{d x^{2}}+(\alpha+2 \beta) x \frac{d y}{d x}-n(\alpha n+2 \beta) y=0
$$

is changed into

$$
\left(u^{2}-1\right) \frac{d^{2} y}{d u^{2}}+(1+2 \gamma) u \frac{d y}{d u}-n(n+2 \gamma) y=0
$$

by putting $u=V \overline{-\alpha} \cdot x, \gamma=\beta / \alpha$. But this is the differential equation satisfied by the generalized Legendre polynomials.

6. It is evident that we can now state the following theorem:

Let

$$
\varphi(y)=a_{0}+a_{1} y+\frac{a_{2}}{2 !} y^{2}+\cdots,
$$

and put.

$$
\varphi\left(2 x z+z^{2}\right)=-P_{0}+P_{1}(x) z+P_{\underline{g}}(x) z^{2}+\cdots
$$

The only cases in which there will be a recursion relation of the form

$$
h(n) P_{n+2}(x)-2 l(n) x P_{n+1}(x)-k(n) P_{n}(x)=0,
$$

where $h(n), l(n)$, and $k(n)$ are polynomials, are essentially where we have the generalized polynomials of Legendre, and the polynomials of Hermite.

INDIANA UNIVERSITY

Bloomington, Ind. 